\title{
Self-referral of Chinese patients with advanced chronic kidney disease and treatment decision-making: A qualitative study
}

\author{
Yajing Gao ${ }^{1}$, Yan Shan ${ }^{1}$, Tingting Jiang ${ }^{2},{\mathrm{Xue} \mathrm{Li}^{1} \text {, Xinxin Jiang }}^{1}$, Li Cai ${ }^{3}$, Sa Xu \\ Tianying Chang ${ }^{2}$, and Hong Wang ${ }^{1}$ \\ ${ }^{1}$ Zhengzhou University \\ ${ }^{2}$ Zhengzhou University First Affiliated Hospital \\ ${ }^{3}$ Shanghai Jiao Tong University School of Medicine Affiliated Renji Hospital
}

November 18, 2020

\begin{abstract}
Abstract: Rationale, aims, and objectives: Chinese patients with advanced chronic kidney disease (CKD), especially rural patients possibly occur self-referral behavior and then treatment decisions followed. It is unclear the relationship between self-referral and treatment decision-making. Thus, the aim of this study was to explore the perceptions and views of self-referral and treatment decision making among patients with advanced chronic kidney disease. Methods: We conducted semi-structured interviews with 26 patients with advanced kidney disease and 12 nephrologists. Interviews were conducted and analyzed thematically until reaching thematic saturation. Results: We identified three themes reflected: 1) self-referral decision making (self-referral motive, barrier to self-referral, seeking for self-referral information); 2) the views and experience of self-referral care (facilitating shared decision making, imposing psychological pressure, feeling about self-referral communication, challenge to staff-patient relationship); 3) treatment decision making (decisional awareness and roles, cost-benefit trade-off and redicision). Conclusions: Our study identified that organizational and demographic factors, self-referral motives worked together at the self-referral decision-making and treatment decision-making when advanced CKD patients facing with healthcare facilities and treatment options. Those findings suggest stakeholders should accelerate the popularization of peritoneal dialysis technology and establish the CKD screening and management systems. For self-referral patients with advanced CKD, our results suggest specialized dialysis transition care to improve quality of communication and soothe patients' negative emotion.
\end{abstract}

\section{Self-referral of Chinese patients with advanced chronic kidney disease and treatment decision- making:}

\section{A qualitative study}

Yajing Gao ${ }^{1}$, Yan Shan ${ }^{2 *}$, Tingting Jiang ${ }^{3},{\mathrm{Xue} \mathrm{Li}^{1} \text {, Xinxin Jiang }}^{1}, \mathrm{Li}, \mathrm{Cai}^{4}$

$\mathrm{Sa}, \mathrm{Xu}^{1}$, Tianying Chang ${ }^{3}$ and Hong Wang ${ }^{1}$

${ }^{1}$ Nursing and health school of Zhengzhou University

${ }^{2}$ Medical school of Zhengzhou University

${ }^{3}$ Zhengzhou University First Affiliated Hospital

${ }^{4}$ Renji Hospital Affiliated to Shanghai Jiao Tong University

*Correspondence: sy110@sina.com

${ }^{2}$ Zhengzhou University 
Full list of author information is available at the end of the article

\begin{abstract}
:
Rationale, aims, and objectives:

Chinese patients with advanced chronic kidney disease (CKD), especially rural patients possibly occur selfreferral behavior and then treatment decisions followed. It is unclear the relationship between self-referral and treatment decision-making. Thus, the aim of this study was to explore the perceptions and views of self-referral and treatment decision making among patients with advanced chronic kidney disease.
\end{abstract}

Methods: We conducted semi-structured interviews with 26 patients with advanced kidney disease and 12 nephrologists. Interviews were conducted and analyzed thematically until reaching thematic saturation.

Results: We identified three themes reflected: 1) self-referral decision making (self-referral motive, barrier to self-referral, seeking for self-referral information); 2) the views and experience of self-referral care (facilitating shared decision making, imposing psychological pressure, feeling about self-referral communication, challenge to staff-patient relationship); 3) treatment decision making (decisional awareness and roles, cost-benefit trade-off and redicision).

Conclusions: Our study identified that organizational and demographic factors, self-referral motives worked together at the self-referral decision-making and treatment decision-making when advanced CKD patients facing with healthcare facilities and treatment options. Those findings suggest stakeholders should accelerate the popularization of peritoneal dialysis technology and establish the CKD screening and management systems. For self-referral patients with advanced CKD, our results suggest specialized dialysis transition care to improve quality of communication and soothe patients' negative emotion.

Keywords: Self-referral, Decision-making, Advanced chronic kidney disease, Qualitative research

\title{
1| INTRODUCTION
}

Chinese chronic kidney disease (CKD) population is constantly on the rise along with increasing number of diabetes and hypertensive patients ${ }^{1,2}$. China Kidney Disease Network (CK-NET) 2015 Annual Data Report showed that the percentage of hospitalized patients with CKD accounted for $4.8 \%$ among chronic patients ${ }^{3}$. If not well detected and managed, CKD may progress to end-stage renal disease (ESRD), at which stage patients have to rely on renal replacement therapy (RRT) to maintain physical functioning. It's predicted that worldwide amount of RRT usage will more than doubled by $2030^{4}$ and each year hundreds of thousands of ESRD patients is newly registered in European and American ${ }^{5}$. Advanced CKD patients with whose eGFR $<30 \mathrm{~mL} / \mathrm{min} / 1.73 \mathrm{~m}^{2}$ and declining are in transitional period and dialysis modality decision has been ranked as their top 10 priorities $^{6,7}$. Not only that, numerous decision points are extended in succession surrounding treatment decisions such as dialysis initiation. In China, the alternative RRT options include in-center hemodialysis (HD), peritoneal dialysis (PD), kidney transplantation as well as conservative kidney management, an acceptable option for older ESRD patients. No matter which treatment decision will affect or even decide patients' lifestyle and the quality of life. Consequence of the ultimate decision determines the necessity of patients involving treatment decision-making progress to implement shared decision making $(\mathrm{SDM})^{8}$. However, previous researches have demonstrated barriers from different perspectives, including predialysis education, communication and cultural factors ${ }^{9-11}$. More recently, one study analyzed residential location played a significant role in treatment decision-making ${ }^{12}$, which may be applicable to status quo of China. In fact, the healthcare system in China is a three-level system, consisting of primary, secondary and tertiary care according to different regions. Besides, the prevalence of CKD in Chinese rural population is relatively high and similar to urban population ${ }^{2,13}$, deepen the association between CKD health service system and uran-rural areas. Global clinical practice guidelines recommend early or timely referral to a predialysis care at least 12 months before patients starting RRT ${ }^{14}$. However, there is evidence that ESRD patients receive dialysis passively without full physical and mental preparation ${ }^{15,16}$. Studies reported over $50 \%$ ESRD patients undergo urgent dialysis or unplanned dialysis, associated with patients' poor prognosis and huge societal healthcare burdens ${ }^{17}$. As the limit of Chinese nephrology medical resource and patients' 
resistance to nephrology preparation, unplanned and crush dialysis are common for ESRD patients ${ }^{18,19}$. On the other hand, self-referral is much more prevalent in the Chinese context of decentralized CKD management 20. Although Chinese multi-tiered medical system encourage referral rather than self-referral ${ }^{20}$, the urgent challenge for self-referral patients with advanced CKD and health care professionals is to conduct RRT decision meetings and make treatment decisions. Understanding how Chinese patients with advanced CKD to achieve self-referral and clarify the relationship between self-referral and treatment decision-making are crucial to nephrology clinical practice and promote policy reform. Previous researched have focused on referral or treatment decision-making independently. Therefore, the aim of this qualitative study was to explore experience and perspectives of advanced CKD patients and healthcare professionals regarding selfreferral and treatment decision-making.

\section{2| METHOD}

\section{1| Study design and participant population}

We conducted a qualitative descriptive study between 5/2019 and 10/2019 at three affiliated hospitals of Zhengzhou University. The three were public hospitals designated by local medical insurance department. We recruited two groups of respondents including a purposive sample of patients with advanced CKD and a convenience sample of nephrologists. The inclusion criteria for advanced CKD patients were 1) aged 18 or older; 2) eGFR $<20 \mathrm{~mL} / \mathrm{min} / 1.73 \mathrm{~m}^{2}$; 3) patient-initiated referral; 4) able to communication in Chinese; Exclusion criteria were :1) acute kidney injury; 2) be on regular dialysis; 3 ) inability to communicate (e.g. cognitive impairment); The inclusion for nephrologists were:1) provision medical services to patients with advanced CKD;2) at least 5 years of clinical experience;

Purposive sampling captured variation in age, self-referral number, CKD stage. All eligible participants invited to interview gave full informed consent. Sampling continued until data saturation, i.e. no new themes were identified in later interviews ${ }^{21}$.

Nephrologists were recruited via telephone or email by study coordinator (Shan, Y). Interested nephrologists agreed to participant this study. Another researcher (Gao, Y J) approached self-referral patients with advanced CKD after first staff-patients meeting about treatment decision. The recruitment strategy was adapted according to suggestion of clinical managers and clinic schedule. Study coordinator obtained all participants' written inform consent and scheduled interviews. Face-to-face interviews were conducted at a private room and each interview lasted approximately $50 \mathrm{~min}$.

\subsection{Data collection}

All researchers have participated in training course of qualitative method. An interview guide (Table 1) focused on the experience of self-referral and treatment decision was developed for the patient interview. Another interview guide (Table 2 )for nephrologists was written and the questions on self-referral care, dialysis decision meeting and the determinants of RRT decision. Both guides were modified until reaching formal guides in accordance with experts' suggestion and feedback. All interviewed were conducted by one researcher (Gao Y J). Participants were interviewed in private room. Due to geographical and temporal restriction, a telephone interview was used for several nephrologists.

\section{3 | Data analysis}

All interviews were recorded and transcribed verbatim into mandarin Chinese. A phenomenological approach was adopted and inductive thematic analysis utilized to analyze the discourses produced by self-referral patients with advanced kidney disease and health professionals ${ }^{22}$. Nvivo 11 software was used to facilitate and manage data ${ }^{23}$. Data were analyzed following these steps: (1) Familiarizing with data, reading repeatedly transcripts and notes to obtain rough understanding and preliminary comment. (2) Generating initial codes, coding interesting features of the data across the entire data set. (3) Searching for themes, collating codes into potential themes. (4) Reviewing themes, checking if the themes work in relation to transcripts. (5) Defining and naming themes, ongoing analysis for refining each theme and overall story. When there were differences in perspectives, all author negotiated and discussed until agreement were reached. Two researchers (Xu S, 
Jiang XX) conducted the initial data coding. The interviewer (Gao YJ) and the research assistant (Li $\mathrm{x}$ ) co-coded for themes. At least, the team discussed each theme and came to a consensus.

\section{3 | RESULT}

\section{1 | Demographics}

\section{Nephrologists}

Twelve nephrologists participated in the study. The sample comprised four chief physician, five associate chief physician and three attending physicians (See table 3). Eight face to face interviews and four telephone interviews were conduct.

\section{Patients}

Twenty-six patients with advanced kidney disease were interviewed (See table 4); eighteen were in CKD 5 stage and twelve were female; seven patients have a college degree or more; Ten participants were working, while others were not working.

Our analysis identified the three themes: 1. Self-referral decision making (Sub-themes: self-referral motives; barriers to self-referral; searching for self-referral information) 2. The experience and views of self-referral care (Sub-themes: facilitating shared decision making; imposing psychological pressure; feeling about selfreferral communication; challenges on staff-patient relationship); 3. Treatment decision making (Sub-themes: decisional awareness and role; cost-benefit trade-off and redecision); Below we will discuss each theme (also depicted in Fig. 1).

\section{2 | Self-referral decision making}

\section{Self-referral motives}

That was acknowledged upward referral would provide patients more opportunities to seek for high-quality health care resource, including system resources and capacity. Higher healthcare facility was the default choice when kidney disease out of control.

"Whether medical facilities or specialists were advanced than primary hospitals. If it's inevitable to adopt replacement, diagnosis and operation should be done accurately and successfully." (P1)

Especially, newly diagnosed ESRD patients, who were at shock and denial stage, have attempted selfreferral to reverse progression of kidney disease. Initial self-referral patients expressed that the strongest consideration was to change the status quo.

"We didn't really believe existing diagnosis and felt a bit chance to bargaining.... We were so indecisive and resist that we didn't know what to do. We pinned hope on referral hospitals which were famous for kidney diseases to initiate dialysis as late as possible."(P5)

Several participants described negative self-referral motives, occurring after definite diagnosis. Self-referral was placebo than health seeking behavior.

"Going to this hospital once each month for monitoring creatinine concentration is mymandatory work while waiting for the dialysis. I know I am at stake... I anticipate the deadline for dialysis." (P13)

Negative self-referral was mainly decided by families while patients didn't show strong rebuttal.

"In fact, I was reluctant to refer to this hospital because it was the same anywhere. However, my son tried to persuade me more than once... I just didn't want to disappoint him." (P9)

\section{Barriers to self-referral}

Participants described self-referral was restricted by various conditions, especially external factors. The most mentioned were distance to referral hospital, economic condition and medical reimbursement. Those unsurmountable factors usually directly leaded to not referral. 
"Self-referral can't be achieved easily for me, because My families had to suspend normal life and ask for leave work. Besides, it was predicted that the medical bills incurred was higher along with lower reimbursement radio. In general, it would take extra medical expenditure and time". (P19)

Another barrier was a lack of general knowledge of CKD, including symptoms, the severity of illness and such on. Eight patients regretted no earlier self-referral.

"If give me another chance, I would go to the top hospitals earlier (cry...). I really didn't know it was going to happen, so did people around me." (P12)

Two nephrologists expressed another piece of insight about barriers they felt to self-referral during clinic consultation. Four nephrologists expressed their expectation of referral timing and renal disease referral system, reflecting systematic barriers.

"Most self-referral patients are at advanced stage, at which phase physicians can't take more measures to delay disease progression reversibly. However, self-referral patients seem too resist to accept the bad news. If patients can turn to higher authority hospitals earlier for detecting causes, we may be able to slow disease progression".(N2)

\section{Searching for self-referral information}

Although self-referral was put forward by patients or their families, medical professionals remained an important source of information.

"We consulted relatives, my cousin, who worked at another hospital. He recommended the Director of Nephrology. Even now, we keep contact with my cousin" (P8)

The internet was another key channel to obtain information, patients reported they searched for a mass of referral information for choosing hospital and preferred physicians before the referral.

"We look up information on the internet. It is the most convenient way to get the most comprehensive information. Ah, the only downside is we can't distinguish the truth from falsehood." (P11)

\subsection{The experience and views of self-referral care}

\section{Facilitating shared decision making}

When it came to self-referral, participants expressed that it provided patients with advanced kidney disease whose residential district haven't widely developed peritoneal dialysis techniques, more options.

"Peritoneal dialysis has been recommended by global nephrologists, However, local hospitals haven't set up peritoneal dialysis service. In fact, more than a few of ESRD patients prefer this modality. If conditions permit, we hope patients can be entitled to PD's benefits, so government does." (N3)

The development of SDM model has actually affected healthcare professionals firstly and physicians have put more emphasis on patients' involvement and treatment satisfaction.

"Unlike many years ago, we have done a lot of training, consisting of patient-centered service philosophy, doctor-patient relationship analysis et c." (N6)

"What moved me was that my doctor asked me details about life, family. Then she talked with other doctors about treatment preference before I put forward the concerns. I felt she understood me." (P21)

\section{Imposing psychological pressure}

It was obviously patients who facing with treatment decisions undertook huge psychological pressure. Patients expressed self-referral have compelled them get into emotional predicament. 
"Self-referral made me perceive huge pressures from economy, job and family relationship. etc. The dilemma then was my family members and I couldn't accept the approaching dialysis, because we have achieved the better treatment by referral." (P18)

The up and down of self-referral progress and unfamiliar hospital environment also aggravated patients' nervousness and restlessness mood.

"We didn't know what to prepare... Many articles of daily use were bought as we used. Most inconvenient of all was accommodation problem for my wife. She had to share a hospital bed with me for saving expense." (P9)

Not only patients, but also nephrologists felt that they were overwhelmed by self-referral patients' high expectations for treatment.

"one self-referral patient told that he turned to our hospital for hearing about its' reputation after turning to hospitals in Beijing and Nanjing. I felt too hard to deal with patients' overconfidence."(N5)

\section{Feelings about self-referral communication}

Self-referral seemed to increase challenges on staff-patient communication because of unfamiliar background and style, gap between communication expectation and busy clinical practice. Patients described that they dealt consultation with physician gingerly, because they didn't quite clear physicians' styles.

"I was dying to communicate with doctors about my illness, However, I didn't know how to organize accurate words. Besides, the biggest problem was I had little chance to talk with my visiting staffs, they were so busy." (P4)

Two physicians reported the numbers of experienced referral affected the predialysis patients' disease informing, while patients described the first dialogue about treatment decision as sudden and expected experience.

"I felt a little hard to communicate with first-time referral patients. You know, dialysis wasn't accepted easily (by patients), maybe second only to cancer, I didn't sure the first-time referral patients' responses... hum, crying, anger..." (N8)

"I have already perceived the real situation, but I didn't want to hear the verdict. However, self-referral has made clinic visit colder for chief physicians' businesslike manner and stiff communication pattern..." (P10)

Different mastery of CKD disease knowledge among patients determined complexity of communication, especially risk communication. Participants reported there was a contradiction between poor mastery of CKD knowledge and an urgent information desire among more self-referral patients.

"I have little concept about CKD or ESRD, because I was diagnosed advanced CKD several month ago, I even don't know what is renal replacement treatment. When doctors talked with me, I felt terrible and confused..." (P13)

One nephrologist noticed that the family members of self-referral advanced CKD patients played crucial role during staff-patient communication.

"Sometimes, the families were main interlocutor when we talked about dialysis or other examination and treatment." (N12)

\section{Challenges on staff-patients relationship}

Nephrologists frankly described that relationships were challenged by distrust and withhold information. One nephrologist described patients approaching dialysis occasionally would disclose the real past medical history for avoiding expensive detections and delaying dialysis, which probably led to not timely diagnosis and treatment. If staff-patients can't coordinate with conflict about treatment protocols, the built relationship would be drained. 
"We gave suggestions for preforming peritoneal dialysis catheterization, However, some patients felt us made a mountain out of molehill. Besides, some referral patients had the thought that after finishing their disease detections, they turned to social acquaintance for help." (N11)

To a certain extent, staff-patient relationship was challenged by rumor or striking news.

"I have heard negative comments from neighbors. Out of protecting our own interest, I performed as a difficult patient." (P6)

\section{4 | Treatment decision-making}

\section{Decisional awareness and role}

Participants reported self-referral stimulated patients' willingness to involve in their own decision-making. As two nephrologists put it.

"Self-referral patients have put more focus on choosing their own dialysis modalities, they had burning requirement for related knowledge and communication. It signified patients' the concept of participating decisions have gradually experienced transformation. We were glad to see it..." (N 1)

Participants indicated that treatment decision-making should be involved by staff-patient-family jointly, not necessarily decided by one side. Self-referral patients reported that they have strong awareness to acquire disease knowledge and choose suitable option lining with their value and preference, but the ultimate decision maker should be whom can take responsibility for decision, usually staffs.

"Patients should have rights to follow or refused physicians' suggestions. However, the prerequisite was that we must have full understanding. However, we were at a professional disadvantage than doctors, thus, we should defer to HCPs for the fateful decision."(P11)

\section{Cost-benefit trade-off and redecision}

Participants reported that the priority setting must take demographic factors into account in decision situation. The most mentioned factors were residence and medical insurance related to self-referral.

"Going to hemodialysis centers three times a week is difficult for me to achieve, because I live in remote rural area, at where rural health clinics aren't equipped with dialysis medicines." (P25)

"The biggest problem was financial burden... at that moment, What I thought was I chose which dialysis" health care cost was less. Due to sharp disease in family income, I have been noticing medical insurance reimbursement policy." (P8)

What's more, priority setting was possible to trigger redecision about downward referral.

"After long deliberation, I thought peritoneal dialysis was the best choice, but I didn't ask doctors to operate peritoneal dialysis catheterization immediately until I learn about available follow-up in the near hospitals." (P12)

\section{4| DISCUSSION}

In this qualitative study of Chinese self-referral patients with advanced CKD and healthcare professionals, we gave self-referral a broader definition, not just out-of-pocket payment referral but patient-initiative referral with the purpose of minimizing the effect of payment pattern of medical insurance. Although participants described perspectives and experience about self-referral and treatment decision respectively, we identified two groups of contradictions pushing forward self-referral and treatment decision-making: primary healthcare facilities and CKD patient medical needs, high expectation for treatment and reality. In particular, our findings suggest self-referral is one of decision points appearing in the trajectory of ESRD patients, which in turn affects patients' treatment decision-making. The mediating role of self-referral on decision making, either positive or negative, is up to the quality of self-referral care. 
Self-referral decision making was initiated by patients or their families and lasted shorter. This is in contrast to physician-referral decision-making, associated with referral policies and primary care physicians' belief ${ }^{24}$. More recently, other qualitative studies have reported self-referral motives, including advanced healthcare facilities, health concern and organizational issues ${ }^{25-27}$. Our study supporting these findings and extend patients' negative self-referral motives, including going through the motions and catering to families. In fact, some of our findings are consistent with the work by Lovell et $\mathrm{al}^{28}$. about the reasons of older patients delaying dialysis. We found if patients' attitude were negative and obscure, their families would play a crucial role to make self-referral decision, align with the theme identified by Chen et al. ${ }^{29}$ that family function of medical seeking and ancillary care.

In our study, participants reported self-referral contributed to the implementation of SDM model in Chinese nephrology field. Although the understanding and perspective of SDM between nephrologists and patients were different, the self-referral exactly stimulated patients' autonomy and initiative to participate in health-related decisions. Those findings counter work form Rise et al. where they found among patients having a contact for self-referral, there were stronger confidence to cope with disease and more active coping strategies $^{30,31}$. On the other hand, our results are consistent with Greer et al, our participants reported lack of suboptimal co-management and asymmetric renal replacement therapy resource among primarysecondary hospitals were fundamental barriers to quality of medical decision-making ${ }^{32}$. On this basis of expanding advanced CKD patients' options, self-referral is an act to overcome the deficiency of objective conditions. Thus, self-referral patients with advanced kidney disease are more likely to initiate subsequent steps of SDM, including information exchanging and value clarification. Evidence suggests that self-referral patients proactively seeking information about treatment ${ }^{33}$. In our study, we found self-referral patients were equipped with higher information literacy.

Among self-referral patients with ESRD and HCPs, the perceived psychological pressure varied, but the same intense. Although previous studies have described advanced CKD patients' high-level perceived distress during decision-making process, we found self-referral placed heavier psychological pressure to staff-patient along with clinic counselling ${ }^{34}$. Those findings complement Hoffmann et al' investigation demonstrated self-referral patients had higher level of health anxiety, associate with indistinguishable disease and extend the concerns of anxiety, such as long wait time and cumbersome dictation procedures ${ }^{35}$. Previous studies demonstrated patients are emotionally fragile, when the expectation and reality of the treatment don't match $^{36}$. In our study, both groups described they weren't quietly sure how to bring high expectation back to reality. Nephrologists indicated self-referral patients or family members half believed their given regimens, which undermines confidence to communicate with patients. As reported by Sun et al ${ }^{37}$, we found those subtle pressure tense staff-patient relationship. Our study suggest that the situation is more complex and creates a vicious cycle due to poor staff-patient communication.

Cost-benefit trade-off, an integral part of priorities setting, was regarded as a value-based process varying according to decisional circumstances. In our study, participants reported top three cost-benefit considerations that include economic cost, physical functioning cost, returning to society cost. Unlike in Victoor's research $^{38}$, participants described great concerns on measuring the benefit of treatment decision for economic evaluation. This finding supports Walker's study about economic consideration underpin dialysis decisions ${ }^{39}$. In fact, stakeholders are trying to balance resource allocation and minimize dialysis cost. Other most talked focuses were long-term implication, such as complication and job. Our findings suggest that the process of trade off can be approximately more or less identified according to age, degree of education, supporting the results in Vivian's study ${ }^{40}$. Our study is also novel in that it suggests cost-benefit trade-off introduces redicision, whether to downward referral and where to continue treatment. The characteristics of lifelong treatment and Chinese practices to downward referral prompt redecision points emerging ${ }^{41}$.

Our findings have important implications for clinical practice and research.

Self-referral plays positive role to patient profile, but appropriate self-referral timing is worth pondering on. The blurred lines between early referral for CKD and hierarchical medical system result in excessive self-referral and late referral. After all, it reflects the lack of CKD knowledge among public, enlightening 
the screening and publicity work need to be strengthened by Chinese primary care professionals. Additionally, the peritoneal dialysis is still in the stage of application and dissemination, not forming a clearly coherent connection. Those findings provide supports to stakeholders in establishment and improvement of nephropathy management.

This research verifies the hypothesis that self-referral has impact on advanced kidney disease patients' decision-making. To meet the flexibly challenges, researchers need to investigate the status of predialysis service and better understand trajectory of start dialysis. Evidence suggest dialysis decision aids incorporated into predialysis education supports primary physician carrying out CKD patients' follow-up and implementing predialysis preparation ${ }^{42}$. On the other hand, renal clinicians should be well-equipped with empathy ability and communication skills instead of completing consultation. As our findings showed, selfreferral patients were vulnerable and sensitive to conversations with their clinicians. However, not hard to see self-referral patients are easy to recover if supervising physicians and nurses handle properly. Dialysis transition unit is worth learning, covering physical and emotional care ${ }^{43}$. We recommend transition clinic at where trained multidisciplinary team provide patient-center care for newly diagnosed ESRD or approaching dialysis patients.

Our study has important limitations. First, the small sample size limited the representativeness of our findings to general nephrologists and patients with advanced kidney disease. Second, the sample included nephrologists from provincial referral hospital, while the primary care professionals weren't included due to distance restrictions. It is likely that there are difference perspectives among primary care professionals and healthcare professionals. Third, we don't conduct follow-up interviews to self-referral patients referring to downward hospitals. Thus, we were able to identified the contrast experiences. Lastly, theme saturation was a relative concept and might change over time, healthcare policy and new RRT technology.

\section{5 | CONCLUSION}

This is the first study to our knowledge to explore the experience and perspectives between nephrologists and patients with advanced CKD about self-referral and treatment decision-making. Our study identified that organizational and demographic factors, self-referral motives worked together at the self-referral decisionmaking and treatment decision-making when advanced CKD patients facing with healthcare facilities and treatment options. Our study suggest stakeholders should accelerate the popularization of peritoneal dialysis technology and establish the CKD screening and management systems. For self-referral patients with advanced CKD, our results suggest specialized dialysis transition care to improve quality of communication and soothe patients' negative emotion.

\section{Abbreviations}

CKD: Chronic kidney disease; eGFR: Estimated glomerular filtration rate; ESRD: End stage renal disease; HD:Hemodialysis; PD: Peritoneal dialysis; CM: Conservative management;

\section{Declarations}

\section{Acknowledgements}

We expressed thanks to study participants who assisted with recruitment.

\section{Authors' contributions}

Research idea, study: Gao YJ; paper and analysis idea: Shan Y; data analysis/interpretation: Li X, Jiang TT. Each author contributed important intellectual content during manuscript drafting or revision and all authors read and approved the final manuscript.

\section{Funding}

This work was supported by Health Commission of Henan Province. (grant number SBGJ2018053)

\section{Availability of data and materials}


The anonymized datasets used and analyzed during the current study are available from the first author on reasonable request.

\section{Ethics approval and consent to participate}

This study has obtained ethics approval from the Ethics Committee of Zhengzhou University (ZZUIRB202049). All participants received a participant information sheet; they subsequently signed an informed consent from prior to participation.

\section{Consent for publication}

Not applicable

\section{Competing interests}

The authors declare that they have no competing interests.

\section{Authors details}

${ }^{1}$ Zhengzhou University

${ }^{2}$ Zhengzhou University First Affiliated Hospital

${ }^{3}$ Renji Hospital Affiliated to Shanghai Jiao Tong University

1. Hill NR, Fatoba ST, Oke JL, et al. Global Prevalence of Chronic Kidney Disease - A Systematic Review and Meta-Analysis. PLoS One.2016;11(7):e0158765.

2. Duan J, Wang C, Liu D, et al. Prevalence and risk factors of chronic kidney disease and diabetic kidney disease in Chinese rural residents: a cross-sectional survey. Sci Rep. 2019;9(1):10408.

3. Zhang L, Zhao MH, Zuo L, et al. China Kidney Disease Network (CK-NET) 2015 Annual Data Report. Kidney Int Suppl (2011).2019;9(1):e1-e81.

4. Liyanage $\mathrm{T}$, Ninomiya $\mathrm{T}$, Jha $\mathrm{V}$, et al. Worldwide access to treatment for end-stage kidney disease: a systematic review. The Lancet.2015;385(9981):1975-1982.

5. Saran R, Li Y, Robinson B, et al. US Renal Data System 2015 Annual Data Report: Epidemiology of Kidney Disease in the United States.Am J Kidney Dis. 2016;67(3 Suppl 1):Svii, S1-305.

6. Manns B, Hemmelgarn B, Lillie E, et al. Setting research priorities for patients on or nearing dialysis. Clin J Am Soc Nephrol.2014;9(10):1813-1821.

7. Scott J, Owen-Smith A, Tonkin-Crine S, et al. Decision-making for people with dementia and advanced kidney disease: a secondary qualitative analysis of interviews from the Conservative Kidney Management Assessment of Practice Patterns Study. BMJ Open.2018;8(11):e022385.

8. Zee J, Zhao J, Subramanian L, et al. Perceptions about the dialysis modality decision process among peritoneal dialysis and in-center hemodialysis patients. BMC Nephrol. 2018;19(1):298.

9. Cassidy BP, Harwood L, Getchell LE, Smith M, Sibbald SL, Moist LM. Educational Support Around Dialysis Modality Decision Making in Patients With Chronic Kidney Disease: Qualitative Study. Can J Kidney Health Dis. 2018;5:2054358118803323.

10. Muscat DM, Kanagaratnam R, Shepherd HL, Sud K, McCaffery K, Webster A. Beyond dialysis decisions: a qualitative exploration of decision-making among culturally and linguistically diverse adults with chronic kidney disease on haemodialysis. BMC Nephrol.2018;19(1):339.

11. Selman LE, Bristowe K, Higginson IJ, Murtagh FEM. The views and experiences of older people with conservatively managed renal failure: a qualitative study of communication, information and decisionmaking. BMC Nephrol. 2019;20(1):38. 
12. Youl P, Morris B, Jenny A, Morstyn L. What factors influence the treatment decisions of women with breast cancer? Does residential location play a role? Rural Remote Health. 2019;19(2):4497.

13. Jiang L, Liang Y, Qiu B, et al. Prevalence of chronic kidney disease in a rural Chinese adult population: the Handan Eye Study. Nephron Clin Pract. 2010;114(4):c295-302.

14. Singh K, Waikar SS, Samal L. Evaluating the feasibility of the KDIGO CKD referral recommendations. BMC Nephrol. 2017;18(1):223.

15. Campbell-Crofts S, Stewart G. How perceived feelings of "wellness" influence the decision-making of people with predialysis chronic kidney disease. J Clin Nurs. 2018;27(7-8):1561-1571.

16. Van den Bosch J, Warren DS, Rutherford PA. Review of predialysis education programs: a need for standardization. Patient Prefer Adherence. 2015;9:1279-1291.

17. Molnar AO, Hiremath S, Brown PA, Akbari A. Risk factors for unplanned and crash dialysis starts: a protocol for a systematic review and meta-analysis. Syst Rev. 2016;5(1):117.

18. Ye H, Yang X, Yi C, et al. Urgent-start peritoneal dialysis for patients with end stage renal disease: a 10-year retrospective study.BMC Nephrol. 2019;20(1):238.

19. Wang C, Fu X, Yang Y, et al. A Comparison between Intermittent Peritoneal Dialysis and Automatic Peritoneal Dialysis on Urgent Peritoneal Dialysis. Am J Nephrol. 2017;45(6):540-548.

20. Feng D, Zhang D, Li B, et al. Does having a usual primary care provider reduce patient self-referrals in rural China's rural multi-tiered medical system? A retrospective study in Qianjiang District, China. BMC Health Serv Res. 2017;17(1):778.

21. Guest G, Namey E, Chen M. A simple method to assess and report thematic saturation in qualitative research. PLoS One.2020;15(5):e0232076.

22. AJ S, E L, C N, L P. Qualitative thematic analysis based on descriptive phenomenology. Nursing Open.2019;Vol.6(No.3):733-739.

23. Zamawe FC. The Implication of Using NVivo Software in Qualitative Data Analysis: Evidence-Based Reflections. Malawi Med J.2015;27(1):13-15.

24. Stocker JK, Cup EHC, Vonk MC, et al. What moves the rheumatologist? Unravelling decision making in the referral of systemic sclerosis patients to health professionals: a qualitative study. Rheumatol Adv Pract. 2018;2(2):rky027.

25. Kraaijvanger Na, Rijpsma Da, Willink La, e, Lucassen Pb, van Leeuwen Hc, Edwards Md. Why patients self-refer to the Emergency Department: A qualitative interview study(Article). Journal of Evaluation in Clinical Practice. 2017;Vol.23(No.3):593-598.

26. Minderhout RNN, Venema P, Vos HMM, Kant J, Bruijnzeels MA, Numans ME. Understanding people who self-referred in an emergency department with primary care problems during office hours: a qualitative interview study at a Daytime General Practice Cooperative in two hospitals in The Hague, The Netherlands. BMJ open. 2019;Vol.9(No.6):e029853.

27. Oslislo S, Heintze C, Schmiedhofer M, Mockel M, Schenk L, Holzinger F. How to decide adequately? Qualitative study of GPs' view on decision-making in self-referred and physician-referred emergency department consultations in Berlin, Germany. BMJ Open.2019;9(4):e026786.

28. Lovell S, Walker RJ, Schollum JB, Marshall MR, McNoe BM, Derrett S. To dialyse or delay: a qualitative study of older New Zealanders' perceptions and experiences of decision-making, with stage 5 chronic kidney disease. BMJ Open. 2017;7(3):e014781.

29. Zhai H, Lavender C, Li C, Wu H, Gong N, Cheng Y. Who decides? Shared decision-making among colorectal cancer surgery patients in China.Support Care Cancer. 2020. 
30. Rise MB, Evensen GH, Moljord IEO, Rø M, Bjørgen D, Eriksen L. How do patients with severe mental diagnosis cope in everyday life - a qualitative study comparing patients' experiences of self-referral inpatient treatment with treatment as usual? BMC Health Services Research. 2014;Vol.14(No.347).

31. Shay LA, Lafata JE. Understanding patient perceptions of shared decision making. Patient Educ Couns. 2014;96(3):295-301.

32. Greer RC, Liu Y, Cavanaugh K, et al. Primary Care Physicians' Perceived Barriers to Nephrology Referral and Co-management of Patients with CKD: a Qualitative Study. J Gen Intern Med.2019;34(7):1228-1235.

33. Clarke M, Lankappa S, Burnett M, Khalifa N, Beer C. Patients with depression who self-refer for transcranial magnetic stimulation treatment: exploratory qualitative study. BJPsych Bull.2018;42(6):243247.

34. Bezerra CIL, Silva BC, Elias RM. Decision-making process in the pre-dialysis CKD patients: do anxiety, stress and depression matter? BMC Nephrol. 2018;19(1):98.

35. Hoffmann D, Rask CU, Hedman-Lagerlof E, Eilenberg T, Frostholm L. Accuracy of self-referral in health anxiety: comparison of patients self-referring to internet-delivered treatment versus patients clinician-referred to face-to-face treatment. BJPsych Open.2019;5(5):e80.

36. Urban-Baeza A, Zarate-Kalfopulos B, Romero-Vargas S, Obil-Chavarria C, Brenes-Rojas L, ReyesSanchez A. Influence of depression symptoms on patient expectations and clinical outcomes in the surgical management of spinal stenosis. J Neurosurg Spine. 2015;22(1):75-79.

37. Sun J, Zhang L, Sun R, et al. Exploring the influence of resiliency on physician trust in patients: An empirical study of Chinese incidents.PLoS One. 2018;13(12):e0207394.

38. Victoor A, Noordman J, Potappel A, Meijers M, Kloek CJJ, de Jong JD. Discussing patients' insurance and out-of-pocket expenses during GPs' consultations. BMC Health Serv Res. 2019;19(1):141.

39. Rachael C Walker 12 KH, Allison Tong 34 4, Suetonia C Palmer 5 , Mark R Marshall 6 , Rachael L Morton 7. The economic considerations of patients and caregivers in choice of dialysis modality. Hemodialysis International. 2016;Vol.20(No.4):634-642.

40. Reckers-Droog V, van Exel J, 2, Brouwer W, 2. Equity Weights for Priority Setting in Healthcare: Severity, Age, or Both? Value in Health. 2019;Vol.22(No.12):1441-1449.

41. Yu W, Li M, Nong X, et al. Practices and attitudes of doctors and patients to downward referral in Shanghai, China. BMJ open.2017; Vol.7(No.4):e012565.

42. Winterbottom AEa, Gavaruzzi Ta, Mooney Ab, et al. Patient acceptability of the yorkshire dialysis decision aid (Yodda) booklet: A prospective non-randomized comparison study across 6 predialysis services(Article). Peritoneal Dialysis International.2016;Vol.36(No.4):374-381.

43. Hercz G. The Trauma of Dialysis Initiation. J Am Soc Nephrol.2017;28(10):2835-2837.

Table 1 Topic guide for patient

\begin{tabular}{ll}
\hline Interview topic & Areas covered \\
\hline Background & Demographic data Previous medical history \\
Self-referral experience & History of present illness Referral numbers \\
& Reason for self-referral Perceived barrier \\
Perception of clinic consultation & Self-referral expectation Self-referral preparation \\
RRT impact & Experience of clinic consultation \\
Decision-making & Understanding of RRT How will RRT affect them \\
& View of involving in treatment decision making \\
& Value and preference Followed decision points \\
\hline
\end{tabular}


Table 2 Topic guide for health care professional

\begin{tabular}{ll}
\hline Interview topic & Areas covered \\
\hline Perception of self-referral & Perspective of patient self-referral: including: . \\
& Difference from physician-initiated referral . \\
& Self-referral timing . How to improve self-referral \\
Self-referral consultation & Experience of self-referral consultation Including: . \\
& Factors influencing the consultation . Barrier to \\
& communication \\
Dialysis decision-making & Attitude to shared decision making How they \\
& communicate with patients regarding decisions \\
& Recommend for RRT modality and consideration \\
\hline
\end{tabular}

Table 3 Characteristics of nephrologists $(n=12)$

\begin{tabular}{llll}
\hline Code & Gender & Years in practice & Specialty \\
\hline N1 & Male & 6 years & Peritoneal dialysis \\
N2 & Female & 10 years & Diabetic nephropathy \\
N3 & Male & 8 years & Hemodialysis \\
N4 & Female & 12 year & Peritoneal dialysis \\
N5 & Male & 23 years & Lupus nephropathy \\
N6 & Male & 20 years & Hemodialysis \\
N7 & Female & 18 years & Peritoneal dialysis \\
N8 & Male & 15 years & Diabetic nephropathy \\
N9 & Male & 10 years & Nephrotic syndrome \\
N10 & Male & 15 years & Peritoneal dialysis \\
N11 & Female & 12 years & Hemodialysis \\
N12 & Male & 26years & Diabetic nephropathy \\
\hline
\end{tabular}

Table 4: Characteristics of patients $(n=26)$

\begin{tabular}{lllll}
\hline Code & Gender & Age & CKD stage & Duration of diagnosed CKD \\
\hline P1 & Female & 31 & 5 & 6 months \\
P2 & Male & 44 & 5 & 2 years \\
P3 & Female & 42 & 5 & 8 months \\
P4 & Male & 60 & 4 & 10 years \\
P5 & Male & 54 & 5 & 7 years \\
P6 & Female & 28 & 5 & 3 years \\
P7 & Male & 36 & 5 & 1 year \\
P8 & Male & 37 & 5 & 2 years \\
P9 & Male & 27 & 5 & 6 months \\
P10 & Male & 33 & 5 & 1.5 years \\
P11 & Female & 56 & 4 & 13 years \\
P12 & Female & 48 & 5 & 5 years \\
P13 & Male & 57 & 5 & 9 years \\
P14 & Male & 30 & 5 & 3 years \\
P15 & Female & 29 & 5 & 8 months \\
P16 & Male & 44 & 5 & 2 years
\end{tabular}




\begin{tabular}{lllll}
\hline Code & Gender & Age & CKD stage & Duration of diagnosed CKD \\
\hline P17 & Male & 53 & 4 & 6 years \\
P18 & Male & 58 & 5 & 5 years \\
P19 & Female & 32 & 5 & 4 months \\
P20 & Female & 55 & 4 & 3 years \\
P21 & Female & 40 & 5 & 2 years \\
P22 & Male & 34 & 4 & 6 months \\
P23 & Female & 47 & 5 & 6 years \\
P24 & Male & 58 & 5 & 11 years \\
P25 & Female & 51 & 4 & 5 months \\
P26 & Female & 37 & 5 & 1 year \\
\hline
\end{tabular}

\section{Hosted file}

figure.pdf available at https://authorea.com/users/376877/articles/493713-self-referral-ofchinese-patients-with-advanced-chronic-kidney-disease-and-treatment-decision-making-aqualitative-study 